\title{
HIGHLIGHTS
}

PREVENTION

\section{Oral bisphosphonate use may protect women from breast cancer}

Bisphosphonates are commonly prescribed for the treatment of osteoporosis and for the prevention of cancer-induced bone loss. Interest in the potential of bisphosphonates for the prevention of cancer was sparked by a publication last year by Michael Gnant and coworkers. They reported that intravenous adjuvant treatment with zoledronic acid in breast cancer patients reduced the risk of disease progression by $36 \%$.

These results were observed with interest by many in the field and at least two research groups have since been working on the significance of these findings in terms of breast cancer prevention. In concurrent publications, teams lead by Rowan Chlebowski and Gad Rennert have reported significant reductions in breast cancer incidence in women taking oral bisphosphonates.

The two groups adopted different strategies to ask the same questions-what effect do oral bisphosphonates have on breast cancer, and is it the treatment itself or the indication requiring treatment that is the cause of any positive effect?

Rennert and colleagues adopted an association study approach, which involved recruiting newly diagnosed breast cancer patients as well as demographically matched heathy controls. Crucially, the team did not rely solely on questionnaire evidence-they also had access to the computerized prescription files.
Rennert described their key findings as: "the use of bisphosphonates... was significantly more common in healthy women than in the women with breast cancer. This suggests that use of bisphosphonates is 'protective' with a protection magnitude of $28 \%$ after correction for a variety of possible confounders." Interestingly, the protective effect of bisphosphonates could only be observed after 1 year, and no cumulative protection was observed thereafter, indicating that it is the use of the drug rather than the indication being treated that is responsible for the observed effect.

Chlebowski and colleagues used the data collected in the observational study The Women's Health Initiative, which includes bone mass density, 5-year hip fracture scores as well as oral bisphosphonate use and breast cancer incidence. The researchers used this rich source of information to attempt to eliminate the effect of a potential confounder-those receiving bisphosphonates usually have a low bone mineral density and this is associated with reduced breast cancer incidence.

The researchers were able to establish an adjustment for potential bone mineral density differences; "after this adjustment, we found women who were bisphosphonate users had 32\% fewer invasive breast cancers than non-usersincluding a trend for fewer hormone

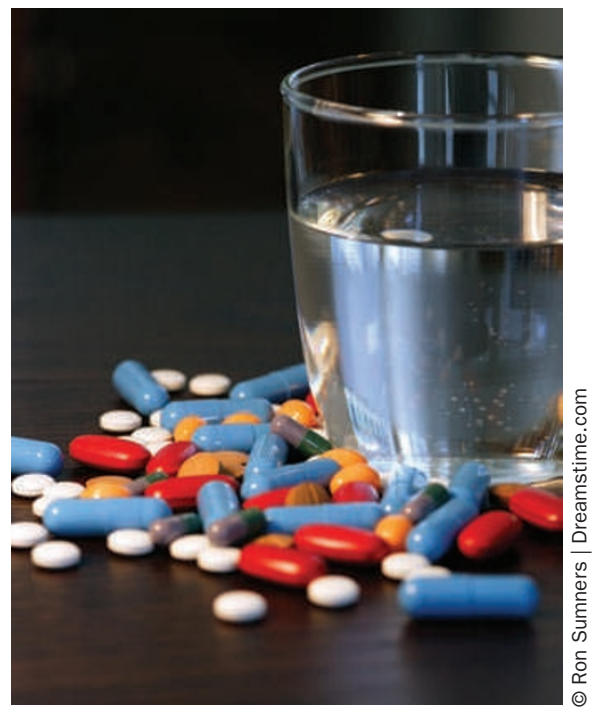

receptor cancers", explained Chlebowski. As about 40 million prescriptions for bisphosphonates are written every year in the USA alone, Chlebowski appropriately pointed out that "there could be a substantial public health benefit" to this finding. Both groups now plan to conduct prospective trials to confirm this discovery in breast cancer and in other indications.

\section{Rebecca Kirk}

Original articles Chlebowski, R. T. et al. Oral
bisphosphonate use and breast cancer incidence in
postmenopausal women. J. Clin. Oncol. 28, 3582-3590
(2010) | Rennert, G. et al. Use of bisphosphonates and
risk of postmenopausal breast cancer. J. Clin. Oncol. 28,
$3577-3581$ (2010)
Further reading Gnant, M. et al. Endocrine therapy plus
zoledronic acid in premenopausal breast cancer. N. Engl. J.
Med. 360,679-691 (2009)

